\title{
The adenylate cyclase gene MaAC is required for virulence and multi-stress tolerance of Metarhizium acridum
}

\author{
Shuyang Liu ${ }^{1,2,3 \dagger}$, Guoxiong Peng ${ }^{1,2,3 \dagger}$ and Yuxian Xia ${ }^{1,2,3^{*}}$
}

\begin{abstract}
Background: The efficacy of entomopathogenic fungi in pest control is mainly affected by various adverse environmental factors, such as heat shock and UV-B radiation, and by responses of the host insect, such as oxidative stress, osmotic stress and fever. In this study, an adenylate cyclase gene ( $M a A C$ ) was cloned from the locust-specific entomopathogenic fungus, Metarhizium acridum, which is homologous to various fungal adenylate cyclase genes. RNA silencing was adapted to analyze the role of MaAC in virulence and tolerance to adverse environmental and host insect factors.

Results: Compared with the wild type, the vegetative growth of the RNAi mutant was decreased in PD (potato dextrose medium), Czapek-dox and PDA plates, respectively, demonstrating that MaAC affected vegetative growth. The CAMP levels were also reduced in PD liquid culture, and exogenous CAMP restored the growth of RNAi mutants. These findings suggested that MaAC is involved in CAMP synthesis. The knockdown of MaAC by RNAi led to a reduction in virulence after injection or topical inoculation. Furthermore, the RNAi mutant grew much slower than the wild type in the haemolymph of locust in vitro and in vivo, thus demonstrating that MaAC affects the virulence of M. acridum via fungal growth inside the host locust. A plate assay indicated that the tolerances of the MaAC RNAi mutant under oxidative stress, osmotic stress, heat shock and UV-B radiation was decreased compared with the wild type.

Conclusion: MaAC is required for virulence and tolerance to oxidative stress, osmotic stress, heat shock and UV-B radiation. MaAC affects fungal virulence via vegetative growth inside the insect and tolerance against oxidative stress, osmotic stress and locust fever.
\end{abstract}

Keywords: Biocontrol agents, Entomopathogenic fungi, Conidia, Virulence, Environmental stress

\section{Background}

Fungal biocontrol agents, which are widespread and environmentally safe, have great potential in integrated pest management. However, the application of entomopathogenic fungi such as Metarhizium acridum in the field has been held back owing to their poor efficacy [1]. During the infection process of entomopathogenic fungi, germ tubes are produced after the fungal conidia attach

\footnotetext{
* Correspondence: yuxianxia@cqu.edu.cn

${ }^{\dagger}$ Equal contributors

${ }^{1}$ Genetic Engineering Research Center, College of Bioengineering,

Chongqing University, Chongqing 400030, P. R. China

${ }^{2}$ Chongqing Engineering Research Center for Fungal Insecticides, Chongqing 400030, P. R. China

Full list of author information is available at the end of the article
}

to the insect cuticle, and then differentiate into swollen infection structures called appressoria. The appressoria produce penetration pegs, which penetrate the host $\mathrm{cu}$ ticle via a combination of mechanical pressure and $\mathrm{cu}-$ ticle degrading enzymes, before piercing the surface of the host into the blood cavity. They produce a large number of hyphae through budding, thereby exhausting the nutrition of the insect host [2]. During the course of fungal infection, pathogenic fungi encounter various adverse factors from the host insect, such as antifungal substances on the cuticle [3], oxidative stress during infection [4], osmotic stress inside the host body [5], behavioral changes, such as locust fever during the early infection stage [6,7], and environmental stresses, including heat shock and UV radiation [8]. Research on the

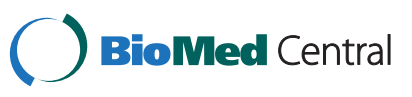


regulatory processes involved in response to adverse factors from the host and environment is essential for the commercial development and improvement of fungi as biocontrol agents.

As major regulators of virulence determinants, the signal transduction pathways of fungal pathogens have been extensively researched. In fungi and yeasts, the cAMP (adenosine 3', 5'-cyclic monophosphate) signaling cascade has been co-opted for a multitude of cellular processes and development. cAMP regulates morphogenesis and virulence in a variety of fungi [9]. Adenylyl cyclase anchored in membrane is responsible for catalyzing the conversion of ATP to cAMP [10]. Recent studies indicate that adenylate cyclase is required for normal vegetative growth, infection structure formation and virulence in phytopathogenic fungi. The role of adenylate cyclase enzymes has been investigated in several fungal species [10-12]. Magnaporthe oryzae depleted of adenylate cyclase (MAC1) was incapable of penetrating the surface of susceptible rice leaves because it could not form appressoria [11]. In the post-harvest necrotrophic fungus Botrytis cinerea, the deletion of the gene encoding adenylate cyclase reduced intracellular cAMP levels, causing delayed vegetative growth, lesion development and in planta sporulation [12]. An adenylate cyclase $(S A C-1)$ deletion mutant in Sclerotinia sclerotiorum exhibited aberrations in sclerotial initiation, possessed altered oxalate levels, and showed reduced virulence due to the lack of infection cushion formation [10]. Targeted disruption of the adenylate cyclase-coding gene in Fusarium proliferatum retarded vegetative growth, increased conidiation and delayed conidial germination [13]. Although adenylate cyclase plays various roles in a number of fungi, the function of adenylate cyclase in entomopathogenic fungi has not been explored up to date.

In this study, we cloned the full-length cDNA of adenylate cyclase from the locust-specific $M$. acridum strain, CQMa 102, designated MaAC. The MaAC transcript level of $M$. acridum was knocked-down by RNAi and the roles of $M a A C$ in pathogenicity and tolerance to stresses were analyzed. Our results showed that MaAC contributed to vegetative growth, virulence and tolerance to various adverse host insect and environmental factors. The results demonstrated that impairment in the virulence of the MaAC RNAi mutant was caused by decreased vegetative growth and tolerance to adverse conditions encountered during host infection.

\section{Results}

\section{Isolation and characteristics of $M a A C$}

A 6,507 bp of cDNA encoding adenylate cyclase $(\mathrm{MaAC})$ was isolated and sequenced (GenBank accession JQ358775). Alignment with the DNA sequence showed that the MaAC gene contained an open reading frame (ORF) and was interrupted by two introns located at the $\mathrm{N}$ terminus ( $955 \mathrm{bp}$ to $1,291 \mathrm{bp}$ ) and the $\mathrm{C}$ terminus (6,219 bp to $6,279 \mathrm{bp})$. The complete ORF of MaAC encoded a predicted protein of 2,169 amino acids (aa) with a molecular mass of $542.0 \mathrm{kDa}$. An analysis using SignalP suggested that the N-terminal sequence of $M a A C$ had no signal peptide. The predicted protein had a high similarity to the adenylate cyclase gene $(A C Y)$ of Metarhizium anisopliae (98\% identity, EFY97222.1), the adenylate cyclase gene of Cordyceps militaris (98\% identity, EGX90508.1), MAC1 of M. oryzae (96\% identity, AAC34139.1) and SAC1 of S. sclerotiorum (95\% identity, ABF71879.1). A fungal phylogenetic tree was established using MEGA 4.0 (Figure 1). MaAC was most similar to the sequence of the entomopathogenic fungus $M$. anisopliae, belonging to the Sordariomycetes. All species were members of the subdivision Pezizomycotina in the division Ascomycota.

\section{Knocked-down MaAC transcription by RNAi}

We conducted an RNA interference (RNAi) strategy to study the function of $M a A C$. Phosphinothricin-resistant transformants of $M$. acridum were generated by transformation with the vector $\mathrm{pK}_{2}-\mathrm{Pb}-\mathrm{MaAC}-\mathrm{RNAi}$ (Figure $2 \mathrm{~A}$ ). To investigate the efficiency of RNAi, the wild type and RNAi mutants of $M a A C$ were analyzed by quantitative RT-PCR. Compared to the wild type, MaAC transcription in the RNAi mutants was downregulated by $66.0 \%, 43.5 \%, 23.1 \%$, $36.2 \%$ and $38.8 \%$, respectively (Figure $2 \mathrm{~B}$ ). These results demonstrated that the transcription of $M a A C$ was efficiently knocked down.

\section{$M a A C$ affects growth in vitro}

The phenotypes of the MaAC RNAi mutants in vitro were analyzed on PDA and Czapek-dox medium (Figure 3A). A variety of morphological abnormalities were observed in the MaAC RNAi mutants. On PDA, the growth of the $M a A C$ RNAi mutants was reduced, mycelium formation was delayed, and the colonies of RNAi mutants were smaller compared to the wild type. On Czapek-dox medium, the conidiation of the MaAC RNAi mutants was also delayed, and the colonies of RNAi mutants were lighter in comparison to the wild type. The AC-RNAi-3 mutant had the most significant difference compared to the wild type, and was used as the MaAC RNAi mutant in the following experiments.

Vegetative growth in vitro was further quantified by assaying the living cells in PD liquid culture by CellTiter $966^{\circledR}$ AQueous One Solution Assay (Figure 3B). In contrast to the wild type, the growth rate of the AC-RNAi-1 mutant was similar to the wild type, while the other four RNA mutants grew conspicuously slowly $(\mathrm{p}<0.01)$. These results indicated that $M a A C$ affects growth in vitro. The correlation coefficient of the relative expression rate and the growth rate was 0.94 , which was highly significant 


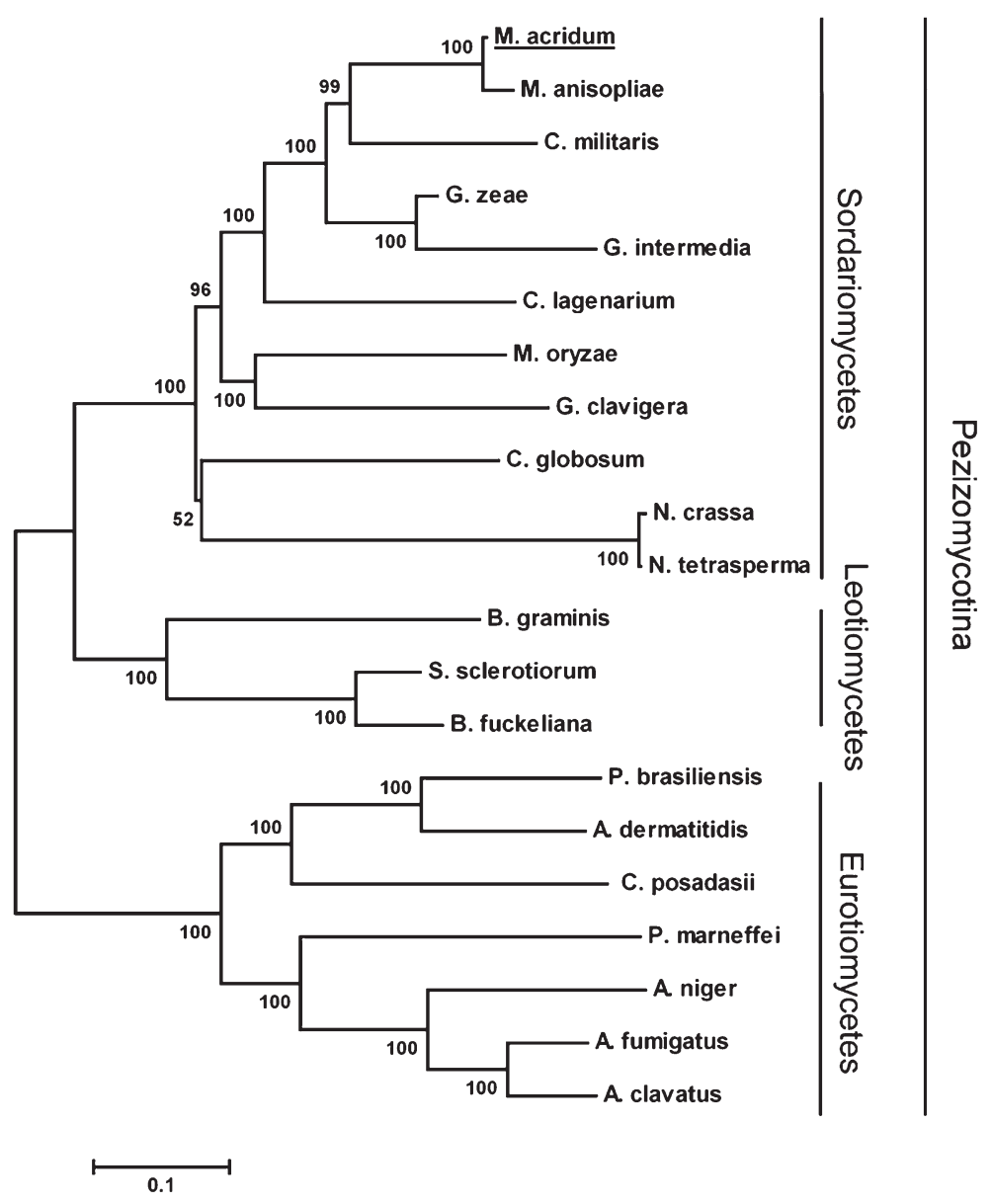

Figure 1 Neighbor-joining tree inferred from MaAC protein sequence alignment. Numbers on the nodes represent the results of bootstrap analyses (1,000 replicates), using the neighbor-joining method. Fungal species: M. acridum (JQ358775), Metarhizium anisopliae (EFY97222.1), Cordyceps militaris (EGX90508.1), Gibberella zeae (XP_381410.1), Gibberella intermedia (AAY79378.1), Colletotrichum lagenarium (BAD04045.1), Magnaporthe oryzae (AAC34139.1), Grosmannia clavigera (EFW99531.1), Chaetomium globosum (XP_001221049.1), Neurospora crassa (BAA00755.1), Neurospora tetrasperma (EGZ77248.1), Blumeria graminis (CAC19663.1), Sclerotinia sclerotiorum (ABF71879.1), Botryotinia fuckeliana (CAB77164.1), Paracoccidioides brasiliensis (AAS01025.1), Ajellomyces dermatitidis (XP_002624019.1), Coccidioides posadasii (EFW21958.1), Penicillium marneffei (XP_002146654.1), Aspergillus niger (XP_001394156.2), Spathaspora passalidarum (EGW29847.1), Aspergillus fumigates (CAC81748.1), Aspergillus clavatus (XP_001268121.1), Spathaspora passalidarum (EGW29847.1).

$(\mathrm{p}<0.01)$. These result showed that the growth rate is related to the relative expression rate of $M a A C$.

\section{MaAC regulates intracellular cAMP levels in $M$. acridum}

As shown in this study, the fungal growth of the $M a A C$ RNAi mutant of $M$. acridum was significantly slower in vitro than that of the wild type. In order to assess whether the growth defect of the RNAi mutant was due to reduced levels of cAMP, we quantified and compared the steady-state levels of cAMP in PD liquid culture. The cAMP level was significantly reduced in the ACRNAi-3 mutant compared to the wild type (Figure 4A) and the cAMP concentration of the MaAC RNAi mutant $(259.4 \mathrm{fMol} / \mathrm{mg})$ was approximately two-fold less than that of the wild type $(486.8 \mathrm{fMol} / \mathrm{mg}$ ) after being cultured for $30 \mathrm{~h}(\mathrm{p}<0.01)$. This demonstrated that
MaAC was involved in cAMP production during the vegetative growth of $M$. acridum. This was further confirmed by the exogenous addition of cAMP (8-Br-cAMP) to the RNAi mutant. As shown in Figure 5, the RNAi mutant grown in the presence of 8 -Br-cAMP showed a great increase in aerial hyphal growth. Thus, exogenous cAMP could restore the growth of the RNAi mutant, which suggested that MaAC was involved in cAMP synthesis.

\section{$M a A C$ is required for in vivo virulence and growth}

Differences in virulence and invasive growth inside insects were also compared between the wild type and RNAi mutant. Figure 6A shows that, 5 days postinoculation on the pronotum, locusts infected by the wild type fungus began to die, while those infected by 

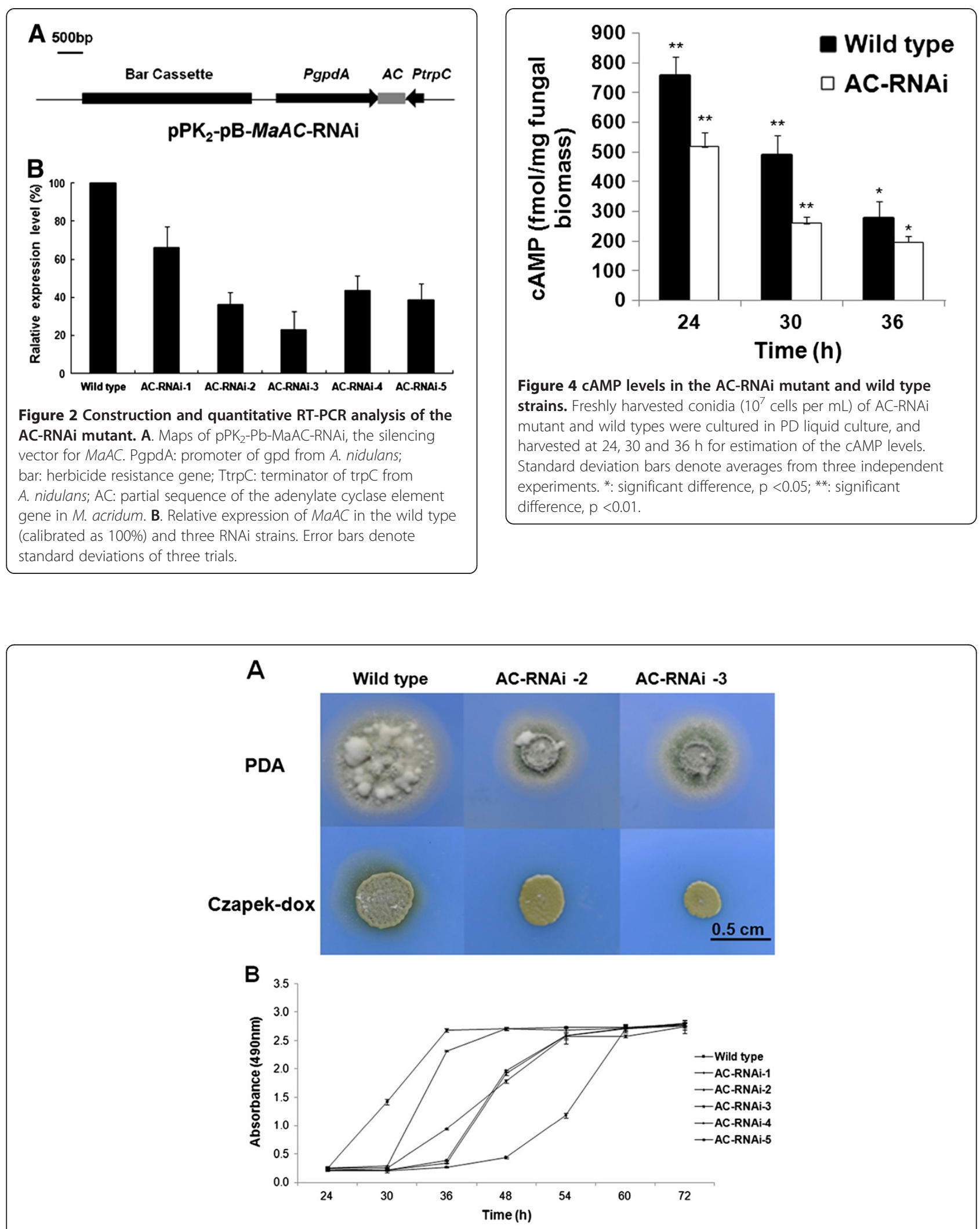

Figure 3 Effect of MaAC on vegetative growth in the wild type and AC-RNAi mutants. A. The colonies were cultured on PDA and Czapek-dox medium for $10 \mathrm{~d}$. Scale bar: $0.5 \mathrm{~cm}$. B. The $\mathrm{OD}_{490}$ after a 3-h incubation of the wild type and AC-RNAi mutant cultured for $72 \mathrm{~h}$ mixed with CellTiter $96^{\circledR}$ AQueous One Solution Reagent in PD liquid culture. Error bars denote the standard deviations from three trials. 




Figure 5 Exogenous addition of 8-Br-cAMP to the AC-RNAi mutant results in increased growth rates. The morphology of the wild type, knockdown control and AC-RNAi mutant colonies grown in the presence of 8-Br-CAMP (5 mM) were inoculated on PDA medium. These cultures were grown for $5 \mathrm{~d}$ prior to documentation. Scale bar: $0.5 \mathrm{~cm}$.

the RNAi mutant died 1 day later. Figure 6B shows that when the insects were inoculated by the injection of conidia into abdominal segments, the locusts began to die 4 days after injection of the wild type, and again the insects treated with the conidia of RNAi mutant died 1 day later. Accordingly, the lethal time value for 50\% mortality $\left(\mathrm{LT}_{50}\right)$ by topical inoculation and injection of the RNAi mutant was significantly higher than that of the wild type $(\mathrm{p}<0.05)$ (Figure 6C), which indicated that $M a A C$ is required for $M$. acridum virulence.

To confirm the effect of $M a A C$ on virulence, fungal growth in vivo was observed by photomicroscopy and quantified by real-time PCR. The $M$. acridum mutant grew significantly more slowly than the wild type (Figure 6E), which was further confirmed by a quantitative assay. Here, the fungal DNA of the wild type was conspicuously higher ( $\sim 4$ times) than that of the RNAi mutant (Figure 6D). Fungal growth cultured in the haemolymph of the locusta in vitro was also observed by photomicroscopy, which showed that the RNAi mutant grew evidently more slowly than the wild type (Figure 6F). Taken together, these results demonstrate that $M a A C$ affects fungal growth both in vivo and in vitro.

\section{MaAC is involved in the tolerance of $M$. acridum to oxidative stress and osmotic stress}

In order to clarify the mechanisms by which MaAC affect the virulence and growth in vivo, the osmosensitivity and $\mathrm{H}_{2} \mathrm{O}_{2}$ tolerance of conidia were analyzed. Firstly, 1/4 SDAY was chosen as a base medium, on which these strains grew with no difference $10 \mathrm{~d}$ postinoculation (Figure 7A). However, RNAi mutants were more sensitive to osmotic stress, and the RNAi mutants colonies were sparse in contrast to the dense ones of the wild type on 1/4 SDAY + KCl (1 M) (Figure 7B). The effect of externally applied $\mathrm{H}_{2} \mathrm{O}_{2}$ on the wild type and RNAi mutants was also tested (Figure $7 \mathrm{C}$ ). The most striking differences between the response of the wild type and RNAi mutants was observed in 1/4 SDAY containing $6 \mathrm{mM} \mathrm{H}_{2} \mathrm{O}_{2}$, where the colonies of the RNAi mutants were sparser than the wild type colonies. These results indicated that $M a A C$ is involved in the tolerance of $M$. acridum to both oxidative and osmotic stresses.

\section{$M a A C$ affects the tolerance to heat and UV light}

The tolerance levels of conidia to heat and UV light were analyzed to clarify the function of $M a A C$. After wet-heat exposure at $45^{\circ} \mathrm{C}$, the germination rate of conidia declined with increasing exposure times, and the conidia germination rates of the wild type strain and mutants appeared to be significantly reduced for each successive 30-min interval (Figure 8A). However, the response to tolerance was obviously different for the wild type strain and RNAi mutant. The conidia germination rate of the wild type strain was higher than that of the mutant. In particular, there was a significant difference at $2 \mathrm{~h}$ and $2.5 \mathrm{~h}(\mathrm{p}<0.01)$. Similar results were observed with the UV-B tolerance test (Figure 8B). Exposure to UV-B for 1-3 h caused a significant difference in the germination rate of conidia between the wild type and RNAi mutant $(\mathrm{p}<0.01)$. These result indicated that the RNAi mutant was more sensitive to UV-B treatment than the wild type. Therefore, $M a A C$ appears to affect the tolerance of $M$. acridum to heat and UV.

\section{Discussion}

Adenylate cyclase regulates a variety of physiological processes in phytopathogenic fungi, including conidiation, conidial germination, vegetative growth, appressoria formation and virulence. In this study, an adenylate cyclase gene, $M a A C$, was identified in a locust-specific 

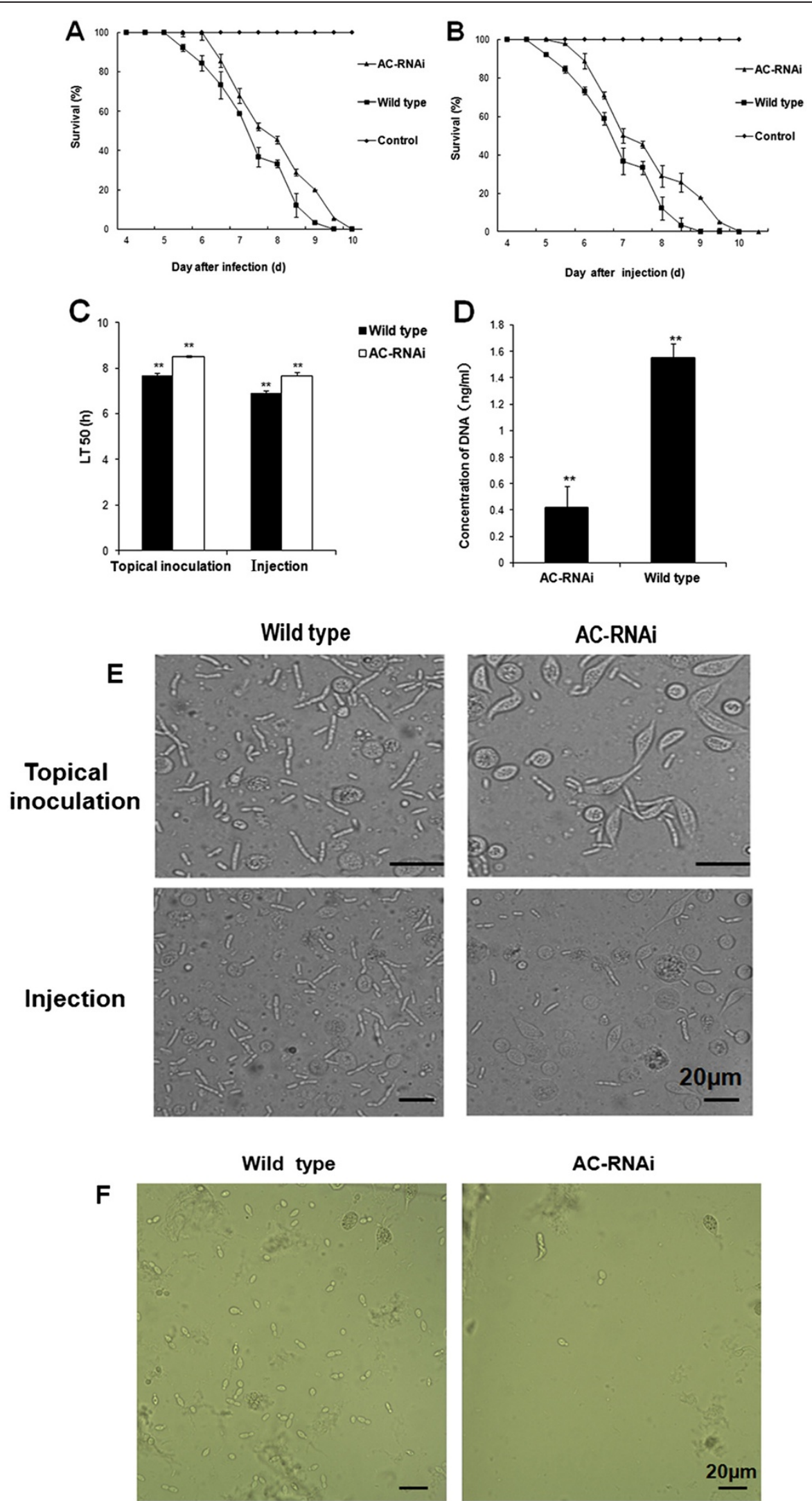

AC-RNAi

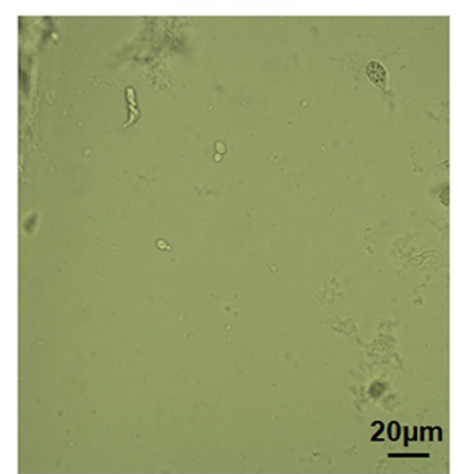

Figure 6 (See legend on next page.) 
(See figure on previous page.)

Figure 6 The virulence and fungal growth in the haemolymph of locust in vivo and in vitro. A. Topical application with $5 \mu \mathrm{L}$ suspensions of $1 \times 10^{7}$ conidia/mL of wild type and RNAi mutant (control insects were inoculated with $5 \mu \mathrm{L}$ cottonseed oil). B. Survival of the locusts by injection with $5 \mu \mathrm{L}$ suspensions of $2 \times 10^{6}$ conidia/mL (control insects were injected with $5 \mu \mathrm{L}$ sterile water). C. Lethal time for $50 \%$ mortality $\left(L T_{50}\right)$ values of Locusta migratoria treated with the wild type or AC-RNAi mutant. Error bars denote standard deviations obtained from five trials. D. DNA concentration of AC-RNAi and wild type in the hemolymph of locusts $48 \mathrm{~h}$ after injection. E. Photomicroscopy of the development of conidiation patterns of M. acridum in the hemolymph of locusts. After $4 \mathrm{~d}$ of infection on the pronotum, the conidiation of the RNAi mutant strain grew slower than the wild type strain. The conidiation of the RNAi mutant strain grew also slower than the wild type strain $3 \mathrm{~d}$ after injection into abdominal segments. F. Photomicroscopy of the development of conidiation patterns of M. acridum in the hemolymph of locusts in vitro. After they were cultured for $24 \mathrm{~h}$, the conidiation of the RNAi mutant strain grew slower than the wild type strain. Scale bar: $20 \mu \mathrm{m}$. Error bars are standard deviations of five trials. *: significant difference, $p<0.05,{ }^{* *}$ : significant difference, $p<0.01$.

entomopathogenic fungus, $M$. acridum. Bioinformatic analysis showed that the cloned $M a A C$ had significant similarity to its homolog from $M$. oryzae and to many other fungal adenylate cyclase genes; the highest degree of similarity was found with the adenylate cyclase of M. anisopliae (98\% identity). The cAMP level of the $M a A C$ RNAi mutant was significantly reduced, and the exogenous addition of cAMP could restore the growth of the RNAi mutant, thus confirming that the MaAC gene encodes adenylate cyclase in $M$. acridum. These results were similar to previous studies on other fungi $[10,12,14]$. Following the deletion of the entire $S A C 1$ coding sequence of S. sclerotiorum [10], cAMP underwent a four-fold reduction in the $S A C 1$ deletion strain compared to the wild type. In $B A C 1$ - and UAC1-defective mutants, intracellular cAMP was detected, which contrasted with the wild type $[13,15]$.

In this report, the downregulation of $M a A C$ led to inhibited growth on in vitro media, including PDA and Czapek-dox medium. In PD liquid culture, it caused

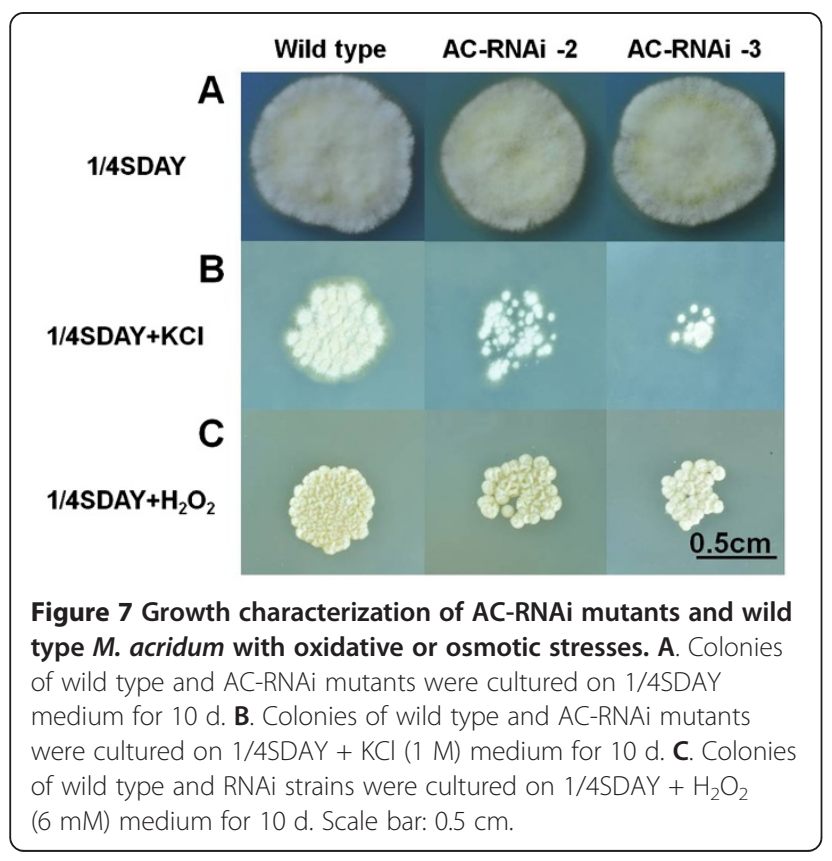

similar effects to previously described adenylate cyclase mutants, such as the SAC1 mutant in S. sclerotiorum [10] and the BAC1 mutant in B. cinerea [12]. Furthermore, $M a A C$ is also involved in the growth of $M$. acridum inside locusts. The virulence of the $M a A C$ mutant was also significantly reduced, thus indicating that $M a A C$ is required for $M$. acridum virulence. This finding is consistent with
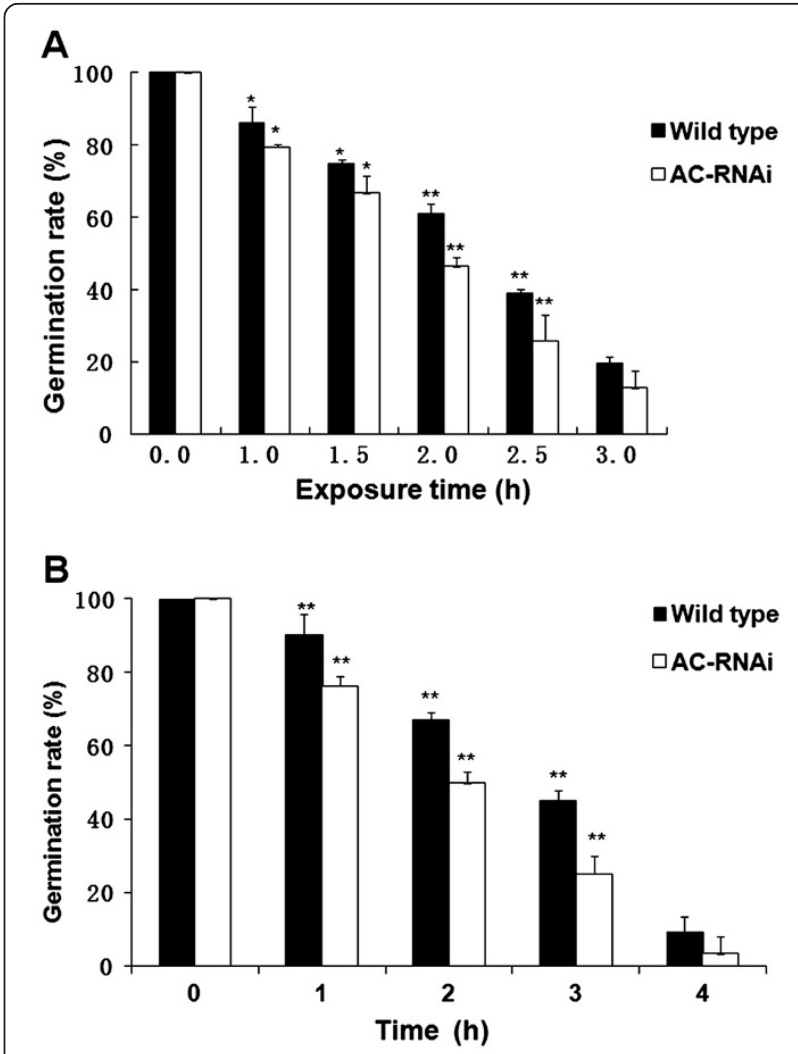

Figure 8 Germination rate of the $M$. acridum wild type and AC-RNAi mutant with wet-heat and UV-B treatments.

A. Germination rate were tested after wet-heat exposure to temperature of $45^{\circ} \mathrm{C}$ for $0,1.0,1.52 .0,2.5$ and 3.0 h. B. Germination rate after UV-radiation exposure for 0, 1, 2, 3 and $4 \mathrm{~h}$. Standard deviation bars denote standard deviations for three independent experiments. *: significant difference, $p<0.05$; **: significant difference, $p<0.01$. 
the role of adenylate cyclase in the virulence of other fungi, including $M$. oryzae [11], B. cinerea [12] and $U$. maydis [15].

Previous research has demonstrated that the tolerance of fungi to stresses such as high temperature [13], UV-B radiation [8,16], oxidative [13] and osmotic stress $[4,5,17]$ is a factor that limits their widespread use. The elevated thermo- and $\mathrm{H}_{2} \mathrm{O}_{2}$-tolerance of the $\Delta$ Fpacy1 mutants indicated that the adenylate cyclase may have negative regulatory roles on the stress response mechanisms of fungal cells [13]. However, the tolerance of the RNAi mutant to the osmotic-, $\mathrm{H}_{2} \mathrm{O}_{2^{-}}$, UV-B and thermal stress was reduced in this study, thus indicating that MaAC may affect the tolerance to multiple stresses through similar regulatory mechanisms in fungal cells.

MaAC affects virulence via controlling the rate of vegetative growth inside the host locust and the tolerance of the fungus to oxidative stress, osmotic stress and locust fever (immune response). The $\Delta \mathrm{LT}_{50}$ values of the AC-RNAi mutant and the wild type after topical inoculation and injection were similar ( $p>0.05)$, but the germination and appressorium formation of the AC-RNAi mutant was not affected (Table 1). The fungal growth of the AC-RNAi mutant in vivo and in vitro was slower compared to the wild type, thus resulting in a reduction of virulence as a result of the slow growth of the ACRNAi mutant in the host body. The effect of adenylate cyclase on virulence is mediated by different mechanisms in different pathogenic fungi. For example, the virulence effect of the $M A C 1$ mutation is due to the inability of the fungus to produce appressoria [11], while the effect of the $B A C 1$ mutation on virulence is due to the absence of sporulation in plants [12]. A fungal pathogen would encounter oxidative stress during infection or osmotic stress inside the host body $[4,5]$, and locust fever (immune response) during the early stage of infection [6,7]. Therefore, the effect of $M a A C$ on stress tolerance in the host insect contributes significantly to the virulence of $M$. acridum.

Table 1 Germination and appressoria formation on locust wings

\begin{tabular}{|c|c|c|c|c|}
\hline & \multicolumn{2}{|c|}{ Germination rate ${ }^{a}(\%)$} & \multicolumn{2}{|c|}{ Appressorium formation rate b $^{b} \%$} \\
\hline & Wild type & AC-RNAi-3 & Wild type & AC-RNAi-3 \\
\hline $14 \mathrm{~h}$ & $33.3 \pm 4.7$ & $25.0 \pm 5.6$ & 0 & 0 \\
\hline $18 \mathrm{~h}$ & $55.7 \pm 4.0$ & $40.3 \pm 1.5$ & 0 & 0 \\
\hline $24 h$ & $80.6 \pm 6.1^{*}$ & $66.3 \pm 6.5^{*}$ & $53.7 \pm 5$ & $48.3 \pm 3$ \\
\hline $28 \mathrm{~h}$ & $99.3 \pm 1.7$ & $98.0 \pm 2.9$ & $79.6 \pm 5$ & $77.6 \pm 4$ \\
\hline
\end{tabular}

a. The germination rate of the wild type and AC-RNAi-3 cultivated on locust wings for $28 \mathrm{~h}$.

b. The appressorium formation rate of the wild type and AC-RNAi-3 cultivated on locust wings for $28 \mathrm{~h}$.

*: Significant difference at a value of $\mathrm{p}<0.05$.

\section{Conclusions}

An adenylate cyclase encoding gene $(M a A C)$ was cloned from the locust-specific entomopathogenic fungus, M. acridum. MaAC affects virulence and fungal growth inside the insect, and is required for its tolerance to oxidative stress, osmotic stress, heat shock and UV-B radiation. MaAC affects fungal virulence via vegetative growth and tolerance to oxidative stress, osmotic stress and locust fever.

\section{Methods}

\section{Strain and culture conditions}

M. acridum strain CQMa102 was isolated from infected yellow-spined bamboo locusts (Ceracris kiangsu Tsai) and was used to derive all strains in this study [18]. The conidia were collected after the fungus was cultured on $1 / 4$ strength Sabouraud's dextrose agar yeast medium (1/4 SDAY; $1 \%$ dextrose, $0.25 \%$ mycological peptone, $2 \%$ agar and $0.5 \%$ yeast extract, $\mathrm{w} / \mathrm{v}$ ) at $28^{\circ} \mathrm{C}$ for $15 \mathrm{~d}$. The medium used for growing mycelia was PD (potato dextrose medium) liquid culture. Czapek-dox medium (3\% saccharose, $0.2 \% \mathrm{NaNO}_{3}, 0.1 \% \mathrm{~K}_{2} \mathrm{HPO}_{4}, 0.05 \% \mathrm{KCl}$, $0.05 \% \mathrm{MgSO}_{4}, 0.001 \% \mathrm{FeSO}_{4}$ ) and potato medium (PDA, 20\% potato, $2 \%$ sucrose, $2 \%$ agar) were used for colony phenotype testing.

\section{Gene cloning, phylogenetic analysis and construction of the MaAC RNAi vector}

Genomic DNA of $M$. acidum was extracted as previously described [19]. The genomic DNA sequence was acquired by PCR with the primer pairs ( $5^{\prime}$-TTCCACGCCAAACCTCAA - $3^{\prime}$ ) and MaAC-R (5'-AGCCAAGTTGTTTCGGTA -3 ') from the whole genomic sequence of $M$. acidum [20]. A full-length cDNA clone of MaAC was amplified using Pyrobest DNA polymerase (TaKaRa, Japan) from a cDNA library of $M$. acridum established in our laboratory [21] with gene-specific primers MaAC-F (5'TTCCACGCCAAACCTCAA -3') and MaAC-R (5'AGCCAAGTTGTTTCGGTA $-3^{\prime}$ ). The resulting PCR product was subcloned into the pMD19-T vector, and transformed into E. coli XL-Blue for determination by GenScript (Nanjing, China). To study the function of $M a A C$, an RNA interference (RNAi) vector was constructed. The partial sequence of $M a A C$ (500 bp) was amplified by MaAC-F (5' - GCGATACACGCCACAAGGACAAAGA-3') and MaAC-R (5'-CCCAAGCTTACTACCAATCTCATCCACCTC-3') from $M$. acridum $M a A C$ cDNA. The resulting PCR product was cloned into pMD19-T (Takara, China) to form pMD19-MaAC. A fragment of $M a A C$ was recovered from pMD19-MaAC by digestion with $E c o$ RI and EcoRV and inserted into the vector $\mathrm{pDPB}$ [22]. The fragment PgpdA-MaAC-PtrpC from $\mathrm{pDPB}$ was inserted at the site between HindIII and XbaI of pPK2-pB $[23,24]$ to form $\mathrm{pPK}_{2}$-pB-MaAC-RNAi. 
Transformation of M. acridum was mediated by Agrobacterium tumefaciens according to the procedure described previously [25]. Transformants were screened on Czapekdox medium containing $80 \mu \mathrm{g} / \mathrm{mL}$ phosphinothricin (PPT) and incubated at $27^{\circ} \mathrm{C}$ for $8 \mathrm{~d}$. Transformants were confirmed by PCR amplification of the RNAi cassette.

\section{Real-time quantitative reverse transcript (qRT-PCR) analysis}

To confirm the expression levels of $M a A C$, the wild type and MaAC-RNAi transformants were grown in PD liquid culture for $2 \mathrm{~d}$ and the mycelia were collected and washed with sterile water. Total RNA was isolated using the SV Total RNA Isolation System (Promega, USA). The synthesis of cDNA and real-time RT-PCR were performed using the method described by Leng et al. [26]. Primers of MaAC-F (5' - GGACGAAGGACTTGACAGACC-3') and MaAC-R (5' -CACAGCATCTCCAGACGAGG-3') were used to detect $M a A C$ expression levels.

\section{Determination of fungal growth}

To characterize the role of $M a A C$ in vegetative growth, the growth rate of the wild type and the RNAi mutants were analyzed using CellTiter $96^{\circledR}$ AQueous One Solution Assay (Promega, USA). In this study, the wild type or RNAi mutants were inoculated in PD liquid culture for 24, 30, 36, 48, 54, 60 and $72 \mathrm{~h}$, respectively. CellTiter $96^{\circledR}$ AQueous One Solution Reagent $(20 \mu \mathrm{L})$ and $100 \mu \mathrm{L}$ culture fluid were directly added to the culture wells, the mixture was incubated for $2 \mathrm{~h}$ at $37^{\circ} \mathrm{C}$, and then the absorbance was recorded at $490 \mathrm{~nm}$ with a 96-well plate reader.

\section{CAMP assay}

The MaAC mutant and the wild type were cultured in PD liquid culture for $36 \mathrm{~h}$. After harvesting, $20 \mathrm{mg}$ mycelia were collected and washed three times with sterile water, followed by treatment with $2 \mathrm{~mL} 0.01 \mathrm{M}$ PBS. Samples were then lyophilized and dissolved in the mixture. They were centrifuged at 4,000 rpm for $20 \mathrm{~min}$ and the supernatant was collected for the test. The cAMP levels were measured with cAMP Enzyme Immunoassay Kit (Sigma, USA), according to the manufacturer's instructions. In total, each assay was repeated three times independently with three biological replicates for every strain.

To test whether exogenous cAMP could restore the growth of RNAi mutant, the cAMP analog, 8-Br-cAMP (Sigma, USA) was added to PDA at a final concentration of $5 \mathrm{mM}$. 8-Br-cAMP (a membrane permeable variant of cAMP) has been extensively used in various studies to artificially cause the enhancement of endogenous cAMP levels [27-29].
Biomass assay and fungal growth in the haemolymph of locust in vivo and in vitro

The virulence of the RNAi mutant and the wild type was tested by topical inoculation and injection into Locusta migratoria adults reared under crowded conditions as previously described by $\mathrm{He}$ et al. [30]. The Locusta migratoria used were all male adult 3 days post-molt. Wild type and RNAi mutants were incubated at $28^{\circ} \mathrm{C}$ on 1/4 SDAY plates for $15 \mathrm{~d}$. Aliquots of $5 \mu \mathrm{L}$ solution of $10^{7}$ conidia/mL of either wild type $M$. acridum or RNAi mutant in cottonseed oil were inoculated on the pronotum. Aliquots of $5 \mu \mathrm{L}$ suspensions $\left(2 \times 10^{6}\right.$ conidia/mL $)$ in sterile water were injected into the hemocoel. Both experiments were repeated five times with 30 insects per replicate. Mortality was recorded every $12 \mathrm{~h}$ after topical inoculation and injection. Mortality was then recorded daily, and lethal time values for 50\% mortality $\left(\mathrm{LT}_{50}\right)$ values were used to estimate the infectivity of M. acridum by DPS software [31]. The growth of $M$. acridum in the host locust was quantified by the detection of fungal rDNA in the infected locust using real-time PCR [32]. After the extraction of $M$. acridum DNA and fungal DNA from the infected locust, fungal DNA was detected by an Icycler iQ Quantitative PCR was performed using specific primers of $M$. acridum: CQMaP-F1: $\quad$ 5'-TGGCATCTTCTGAGTGGTG-3' and CQMaP-R1: 5'-CCCGTTGCGAGTGAGTTA- 3'. To test the fungal growth in the haemolymph of locust in vitro, $50 \mu \mathrm{L}$ of a conidial suspension $\left(1 \times 10^{7}\right.$ conidia/ $\mathrm{mL}$ ) was inoculated into $950 \mu \mathrm{L}$ of locust haemolymph, and the growth of the wild type and mutant was detected $24 \mathrm{~h}$ post inoculation.

\section{Germination and appressoria formation against insect cuticles}

The percentage of germination of wild type and RNAi mutant were measured as described by Liu et al. [18]. The appressorium formation rates were determined from 300 conidia after an $18 \mathrm{~h}$ induction on locust hind wings according to He and Xia [33]. The assay was replicated at least three times.

\section{Oxidative stress, osmotic stress, heat shock and UV-B treatment test}

Growth characterization of the wild type and RNAi mutants were carried out on 1/4 SDAY supplemented with $\mathrm{H}_{2} \mathrm{O}_{2}(6 \mathrm{mM})$ or $\mathrm{KCl}(1 \mathrm{M})$. Samples of conidial suspensions $\left(2 \mu \mathrm{L} ; 5 \times 10^{5}\right.$ conidia/mL) were spotted on each Petri dish and the plates were incubated at $28^{\circ} \mathrm{C}$ for $10 \mathrm{~d}$. The tolerance to wet-heat shock and UV-B treatment of the RNAi mutant and wild type was analyzed as described by Liu et al. [22]. 


\section{Abbreviations}

UV-B: Ultraviolet-B radiation; RNAi: RNA interference; 1/4SDAY: 1/4 strength Sabouraud's dextrose agar medium; PD: Potato dextrose medium; PDA: Potato dextrose agar medium; PBS: Phosphate Buffered Saline; $\mathrm{LT}_{50}$ : Lethal time of 50\%; DPS: Data Processing Standards.

\section{Competing interests}

The authors declare that they have no competing interests.

\section{Authors' contributions}

YX designed the research; SL and GP performed the experiments; SL, GP and $Y X$ wrote the manuscript. All authors read and approved the final version of the manuscript.

\section{Acknowledgments}

This work was supported by a grant from the National Natural Science Foundation of China (No. 30771446) and High Technology Research and Development Program (863) of China (No. 2011AA10A204).

\section{Author details}

${ }^{1}$ Genetic Engineering Research Center, College of Bioengineering, Chongqing University, Chongqing 400030, P. R. China. ${ }^{2}$ Chongqing Engineering Research Center for Fungal Insecticides, Chongqing 400030, P. R. China. ${ }^{3}$ Key Lab of Functional Gene and Regulation Technologies under Chongqing Municipal Education Commission, Chongqing 400030, P. R. China.

Received: 14 February 2012 Accepted: 26 July 2012

Published: 1 August 2012

\section{References}

1. St Leger RJ, Joshi L, Bidochka MJ, Roberts DW: Construction of an improved mycoinsecticide overexpressing a toxic protease. Proc Natl Acad Sci 1996, 93:6349-6354.

2. Weiguo F, Monica P, Sibao W, St Leger R: Protein kinase A regulates production of pathogenicity determinants by the entomopathogenic fungus, Metarhizium anisopliae. Fungal Genet Biol 2009, 46:277-285.

3. Charnley AK, St Leger RJ: The role of cuticle-degrading enzymes in fungal pathogenesis in insects. New York: Plenum Press; 1991:267-287.

4. Yueqing C, Min L, Yuxian X: Mapmi gene contributes to stress tolerance and pathogenicity of the entomopathogenic fungus, Metarhizium acridum. J Invertebr Pathol 2011, 108:7-12

5. Wang CS, Duan ZB, St Leger RJ: MOS1 osmosensor of Metarhizium anisopliae is required for adaptation to insect host hemolymph. Eukaryot Cell 2008, 7:302-309.

6. Inglis GD, Johnson DL, Goettel MS: Effects of temperature and thermoregulation on mycosis by Beauveria bassiana in grasshoppers. Biol Contr 1996, 7:131-139.

7. Lock GD, Pickering SG, Charnley AK: Application of infrared thermography to the study of behavioural fever in the desert locust. J Therm Biol 2011, 36:443-451.

8. Rangel DEN, Braga GUL, Flint SD, Anderson AJ, Roberts DW: Variations in UV-B tolerance and germination speed of Metarhizium anisopliae conidia produced on insects and artificial substrates. J Invertebr Pathol 2004, 87:77-83.

9. Lee N, D'Souza CA, Kronstadt JW: Of smuts, mildews, and blights: cAMP signaling in phytopathogenic fungi. Annu Rev Phytopathol 2003, 41:399-427.

10. Wayne MJ, JeVrey AR: Deletion of the adenylate cyclase (sac1) gene aVects multiple developmental pathways and pathogenicity in Sclerotinia sclerotiorum. Fungal Genet Biol 2007, 44:521-530.

11. Choi W, Dean RA: The adenylate cyclase gene MAC1 of Magnaporthe grisea controls appressorium formation and other aspects of growth and development. Plant Cell 1997, 9:1973-83.

12. Klimpel A, Gronover CS, Williamson B, Stewart JA, Tudzinski B: The adenylate cyclase $(B A C)$ in Botrytis cinerea is required for full pathogenicity. Mol Plant Pathol 2002, 3:439-450

13. Gábor K, Brigitta O, Attila LÁ, García-Martínez J, Hornok L: Adenylyl cyclase regulates heavy metal sensitivity, bikaverin production and plant tissue colonization in Fusarium proliferatum. J Basic Microbiol 2010, 50:59-71.
14. Gold SE, Duncan GA, Barret KJ, Kronstad JW: cAMP regulates morphogenesis in the fungal pathogen Ustilago maydis. Genes Dev 1994 8:2805-2816.

15. Alfredo DM, Jose R, Claudia GL, Scott EG: MAP Kinase and cAMP Signaling Pathways Modulate the $\mathrm{pH}$-Induced Yeast-to-Mycelium Dimorphic Transition in the Corn Smut Fungus Ustilago maydis. Curr Microbiol 2004 49:274-281.

16. Braga GUL, Flint SD, Messias CL, Anderson AJ, Roberts DW: Effect of UV-B on conidia and germlings of the entomopathogenic hyphomycete Metarhizium anisopliae. Mycol Res 2001, 105:874-882.

17. Hallsworth JE, Magan $\mathrm{N}$ : Effects of $\mathrm{KCl}$ concentration on accumulation of acyclic sugar alcohols and trehalose in conidia of three entomopathogenic fungi. Lett Appl Microbiol 1994, 18:8-11.

18. Peng G, Wang Z, Yin Y, Zeng D, Xia Y: Field trials of Metarhizium anisopliae var. acridum (Ascomycota: Hypocreales) against oriental migratory locusts, Locusta migratoria manilensis (Meyen) in Northern China. Crop Prot 2008, 27:1244-1250.

19. Reader U, Broda P: Rapid preparation of DNA from filamentous fungi. Lett Appl Microbiol 1985, 1:17-20.

20. Qiang G, Kai J, Sheng-Hua Y, Yongjun Z, Guohua X, Yanfang S, Zhibing D, Xiao H, Xue-Qin X, Gang Z, Guoxiong P, Zhibing L, Wei H, Bing W, Weiguo F, Sibao W, Yi Z, Li-Jun M, Raymond J, St L, Guo-Ping Z, Yan P, Ming-Guang F, Yuxian X, Chengshu W: Genome Sequencing and Comparative Transcriptomics of the Model Entomopathogenic Fungi Metarhizium anisopliae and M. acridum. PLoS Genet 2011, 7:1

21. Zhang C, Xia Y: Identification of genes differentially expressed in vivo by Metarhizium anisopliae in the hemolymph of Locusta migratoria using suppression subtractive hybridization. Curr Genet 2009, 55:399-407.

22. Liu J, Cao Y, Xia Y: Mmc, a gene involved in microcycle conidiation of the entomopathogenic fungus Metarhizium anisopliae. J Invertebr Pathol 2010, 105:132-138.

23. Hervás-Aguilar A, Rodríguez JM, Tilburn J, Arst HN Jr, Peñalva MA: Evidence for the direct involvement of the proteasome in the proteolytic processing of the Aspergillus nidulans zinc finger transcription factor PacC. J Biol Chem 2007, 282:34735-34747.

24. Lodi T, Fontanesi F, Guiard B: Co-ordinate regulation of lactate metabolism genes in yeast: the role of the lactate permease gene JEN1. Mol Genet Genomics 2002, 266:838-847.

25. Fang $W$, Zhang $Y$, Yang $X$, Zheng $X$, Duan H, Li Y, Pei Y: Agrobacterium tumefaciens-mediated transformation of Beauveria bassiana using an herbicide resistance gene as a selection marker. J Invertebr Pathol 2004, 85:18-24

26. Leng $Y$, Peng $G$, Cao $Y$, Xia Y: Genetically altering the expression of neutral trehalase gene affects conidiospore thermotolerance of the entomopathogenic fungus Metarhizium acridum. BMC Microbio/ 2011, $11: 32$

27. Hao L, Angayarkanni S, Willard FS, Siderovski DP, Shen L, Naqvi NI: Rgs1 regulates multiple Galpha subunits in Magnaporthe pathogenesis, asexual growth and thigmotropism. EMBO J 2007, 26:690-700.

28. Skamnioti P, Gurr SJ: Magnaporthe grisea cutinase 2 mediates appressorium differentiation and host penetration and is required for full virulence. Plant Cell 2007, 19:2674-2689.

29. Ravikrishna R, Naqvi NI: PdeH, a High-Affinity cAMP Phosphodiesterase, Is a Key Regulator of Asexual and Pathogenic Differentiation in Magnaporthe oryzae. PLoS Pathog 2010, 6:5

30. He ZB, Cao YQ, Yin YP, Wang ZK, Chen B, Peng GX, Xia YX: Role of hunchback in segment patterning of Locusta migratoria manilensis revealed by parental RNAi. Dev Growth Differ 2006, 48:439-445.

31. Tang QY, Feng MG: DPS Data Processing System for Practical Analysis. Beijing: Science Press; 2002:1-648.

32. Peng G, Xia Y: The mechanism of the mycoinsecticide diluents on the efficacy of the oil formulation of insecticidal fungus. BioControl 2011 56:893-902

33. He M, Xia Y: Construction and analysis of a normalized cDNA library from Metarhizium anisopliae var. acridum germinating and differentiating on Locusta migratoria wings. FEMS Microbiol Lett 2009, 291:127-135.

doi:10.1186/1471-2180-12-163

Cite this article as: Liu et al:: The adenylate cyclase gene MaAC is required for virulence and multi-stress tolerance of Metarhizium acridum. BMC Microbiology 2012 12:163. 